\title{
FGF signaling network in the gastrointestinal tract (Review)
}

\author{
MASUKO KATOH $^{1}$ and MASARU KATOH ${ }^{2}$ \\ ${ }^{1}$ M\&M Medical BioInformatics, Hongo 113-0033; ${ }^{2}$ Genetics and Cell Biology Section, \\ National Cancer Center Research Institute, Tokyo 104-0045, Japan
}

Received March 29, 2006; Accepted May 2, 2006

\begin{abstract}
Fibroblast growth factor (FGF) signals are transduced through FGF receptors (FGFRs) and FRS2/FRS3SHP2 (PTPN11)-GRB2 docking protein complex to SOSRAS-RAF-MAPKK-MAPK signaling cascade and GAB1/ GAB2-PI3K-PDK-AKT/aPKC signaling cascade. The RAS MAPK signaling cascade is implicated in cell growth and differentiation, the PI3K AKT signaling cascade in cell survival and cell fate determination, and the PI3K aPKC signaling cascade in cell polarity control. FGF18, FGF20 and SPRY4 are potent targets of the canonical WNT signaling pathway in the gastrointestinal tract. SPRY4 is the FGF signaling inhibitor functioning as negative feedback apparatus for the WNT/FGF-dependent epithelial proliferation. Recombinant FGF7 and FGF20 proteins are applicable for treatment of chemotherapy/radiation-induced mucosal injury, while recombinant FGF2 protein and FGF4 expression vector are applicable for therapeutic angiogenesis. Helicobacter pylori, a causative pathogen for peptic ulcer diseases, chronic atrophic gastritis and gastric cancer, injects bacterial proteins into gastric epithelial cells by using Type IV secretion system, which leads to FGF signaling activation through FGF2 upregulation as well as CagA-dependent SHP2 activation. FGFR2 gene is preferentially amplified and overexpressed in diffuse-type gastric cancer. PD173074 is a small-molecule inhibitor for FGFR, while RO4396686 and SU6668 are small-molecule inhibitors for FGFR and other tyrosine kinases. Cocktail therapy using multiple protein kinase inhibitors could enhance the therapeutic effects for gastrointestinal cancer through the reduction of recurrence associated with somatic mutations of drug-target genes. Single nucleotide polymorphism (SNP) and copy number polymorphism (CNP) of genes encoding FGF signaling molecules will be identified as novel risk factors of gastrointestinal cancer. Personalized prevention and personalized medicine based on the combination of genetic screening and novel therapeutic agents could dramatically improve the prognosis of cancer patients.
\end{abstract}

Correspondence to: Dr Masaru Katoh, Genetics and Cell Biology Section, National Cancer Center Research Institute, Tsukiji 5-chome, Chuo-ku, Tokyo 104-0045, Japan

E-mail: mkatoh@ncc.go.jp

Key words: FGF, stem cell biology, gastric cancer, Helicobacter pylori, colorectal cancer, WNT, SNP, systems medicine

\section{Contents}

1. Introduction

2. FGF family

3. Regulation of FGF signaling by WNT

4. FGF signaling network in the stomach

5. FGF signaling network in the colon

6. Clinical application of FGF

7. Clinical application of FGF signaling inhibitors

8. Perspectives

\section{Introduction}

Fibroblast growth factor (FGF) family proteins play key roles in growth and survival of stem cells during embryogenesis, tissues regeneration, and carcinogenesis (1-4). FGF signals are transduced through FGF receptors (FGFRs) and FRS2/FRS3SHP2-GRB2 docking protein complex to SOS-RAS-RAFMAPKK-MAPK signaling cascade and GAB1/GAB2-PI3KPDK-AKT/aPKC signaling cascade (Fig. 1). The RAS MAPK signaling cascade is implicated in cell growth and differentiation, the PI3K $\sim \mathrm{AKT}$ signaling cascade in cell survival and cell fate determination, and the PI3K aPKC signaling cascade in cell polarity control. Here, recent progress, especially in the fields of evolutionary developmental biology, signaling network and clinical applications on FGF research will be reviewed.

\section{FGF family}

FGF1 (aFGF), FGF2 (bFGF), FGF3 (INT2), FGF4, FGF5, FGF6, FGF7 (KGF), FGF8 (AIGF), FGF9, FGF10, FGF11, FGF12, FGF13, FGF14, FGF16, FGF17, FGF18, FGF19, FGF20, FGF21, FGF22 and FGF23 are human FGF members, while Fgf1, Fgf2, Fgf3, Fgf4, Fgf5, Fgf6, Fgf7, Fgf8, Fgf9, Fgf10, Fgf11, Fgf12, Fgf13, Fgf14, Fgf15, Fgf16, Fgf17, Fgf18, Fgf20, Fgf21, Fgf22 and Fgf23 are mouse Fgf family members (1). In 2003, we demonstrated mouse Fgf15 as the ortholog of human FGF19 based on the inter-species comparative genomics analyses on the CCND1-ORAOVI-FGF19FGF4 locus (5). Therefore, 22 FGF family orthologs are conserved between human and mouse genomes.

The zebrafish fgf family consists of fgf1, fgf 2 , fgf3, fgf 4 , fgf5, fgf6a, fgf6b, fgf7, fgf8, fgf9, fgf10, fgf11, fgf12, fgf13, fgf14, fgf16, fgf17a, fgf17b, fgf18a, fgf18b, fgf19, fgf20a, fgf20b, fgf21, fgf22, fgf 23 , fgf 24 and fgf25. Zebrafish 

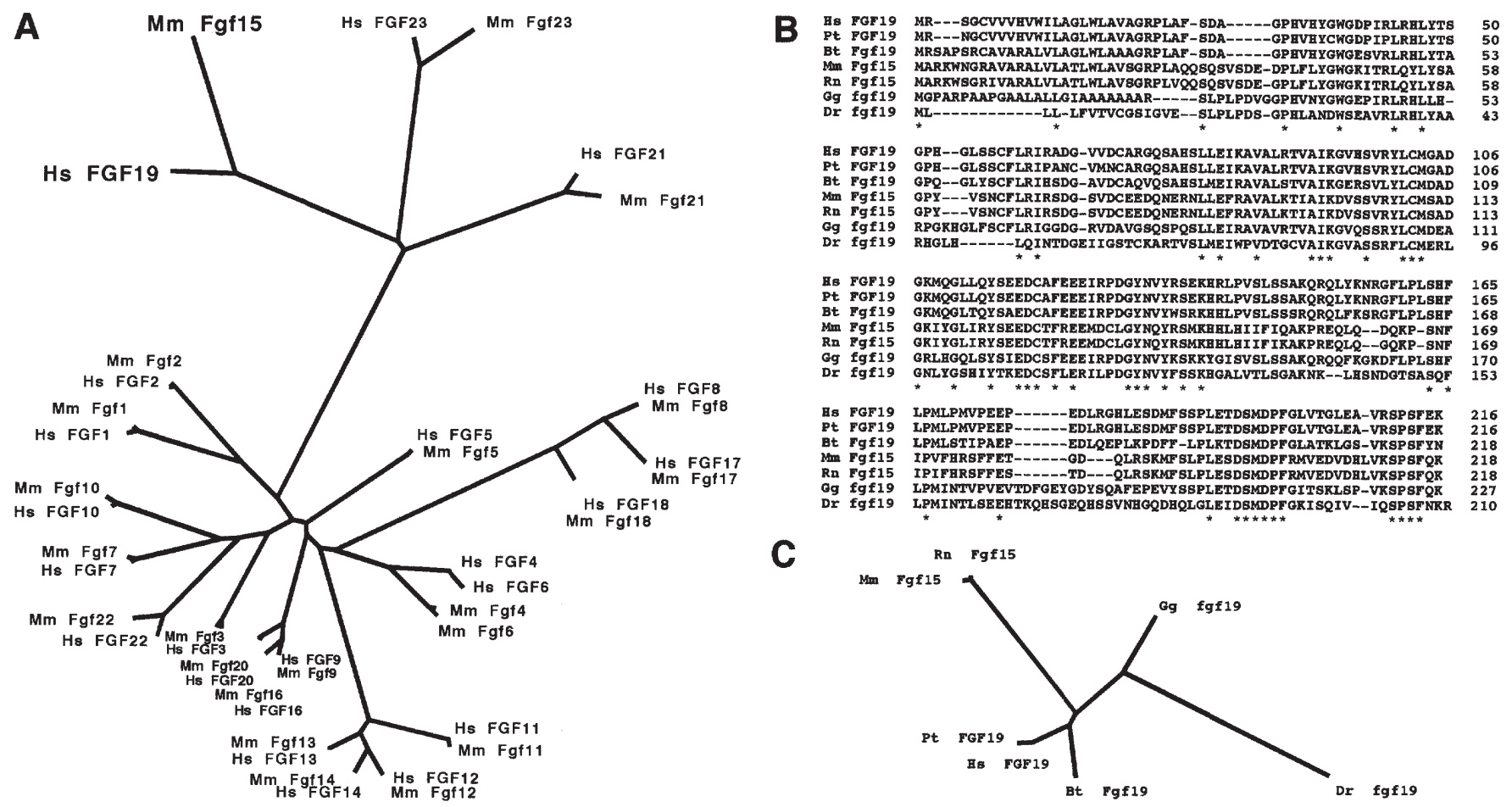

Figure 1. Comparative proteomics on the FGF family. Hs, human; Pt, chimpanzee; Bt, cow; Mm, mouse; Rn, rat; Gg, chicken; Dr, zebrafish. (A), Phylogenetic analysis on human and mouse FGF family members. Human FGF19 and mouse Fgf15 are orthologs; however, FGF19 and Fgf15 are significantly divergent. (B), Alignment of vertebrate FGF19 orthologs. (C), Phylogenetic analysis on vertebrate FGF19 orthologs.

fgf24 and fgf25 are homologs of human FGF8 and FGF10, respectively $(6,7)$. At least $28 f g f$ family genes exist within the zebrafish genome. Zebrafish $f g f 24$ and $f g f 25$ genes are fish specific $f g f$ family members generated due to fish-specific genome duplication.

Phylogenetic analyses on human and mouse FGF family members showed that human FGF19 (NP_005108.1) and mouse Fgf15 (NP_032029.1) were significantly divergent (Fig. 1A). We previously identified and characterized rat Fgf15 and zebrafish fgf19. In this study, we identified chimpanzee FGF19 and cow Fgf19 genes within NW_113926.1 and NW_930789.1 genome sequences, respectively. Complete CDS of chimpanzee FGF19 and cow Fgf19 were determined by assembling exonic regions to determine amino-acid sequence of chimpanzee FGF19 and cow Fgf19 (Fig. 1B). Phylogenetic analyses on vertebrate FGF19 orthologs next revealed that cow Fgf19 was more homologous to primate FGF19 and chicken fgf19 than to rodent Fgf15 (Fig. 1C). These facts indicate that significant nucleotide substitutions within the Fgf19 locus occurred specifically in the rodent lineages to induce functional divergence of rodent Fgf19 orthologs (Fgf15) from other mammalian FGF19 orthologs.

\section{Regulation of FGF signaling by WNT}

FGF signaling pathway networks with WNT signaling pathway during a variety of cellular processes, such as early embryogenesis, body-axis formation, limb-bud formation, neurogenesis, hematogenesis, hepatogenesis, gastrointestinal morphogenesis, MMTV-induced mammary carcinogenesis, and human colorectal carcinogenesis $(1,2,8,9)$.
We have comprehensively cloned (or identified) and characterized WNT signaling molecules within the human genome, because WNT signaling molecules implicated in carcinogenesis and embryogenesis are pharmacogenomics targets in the field of oncology and regenerative medicine (http://www.esi-topics.com/fmf/2005/september05-Masaru Katoh.html). WNT signals are transcuced through Frizzled (FZD) seven-transmembrane-type receptors, LRP5, LRP6, ROR1, ROR2, and RKY. WNT1, WNT2, WNT2B, WNT3, WNT3A, WNT8A, WNT8B, WNT10A, and WNT10B are canonical WNTs to activate the $\beta$-catenin pathway. Canonical WNT signals are transduced to the transcriptional complex consisting of TCF/LEF, B-catenin, BCL9/BCL9L and PYGO1/ PYGO2 to activate transcription of target genes (10-21). We have reported that FGF16, FGF 18 and FGF20 genes are evolutionarily conserved targets of the canonical WNT signaling pathway based on the comparative genomics on the 5 '-promoter regions of the $F G F$ family genes (22-24).

Three TCF/LEF-binding sites were identified at about $1400 \mathrm{bp}, 1250 \mathrm{bp}$ and $200 \mathrm{bp}$ upstream of the transcriptional start site of the human FGF7 gene (Fig. 2A and B). Chimpanzee $F G F 7$ promoter, cow $F g f 7$ promoter, mouse $F g f 7$ promoter and rat $F g f 7$ promoter were then identified within NW_116403.1, AC161632.2, AL845292.4 and AC130100.3 genome sequences, respectively, by using the BLAST programs as described previously (25-30). Phylogenetic analysis revealed that cow $\mathrm{Fgf7}$ promoter was more related to primate $F G F 7$ promoters than to rodent $F g f 7$ promoters (Fig. 2C). Three TCF/LEFbinding sites within human $F G F 7$ promoter were conserved in the chimpanzee $F G F 7$ promoter (Fig. 2D). The most proximal TCF/LEF-binding site within human FGF7 
A

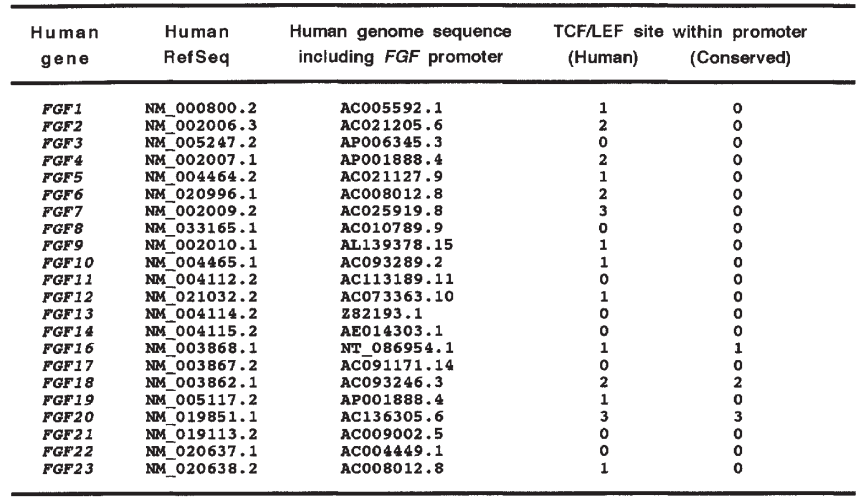

C

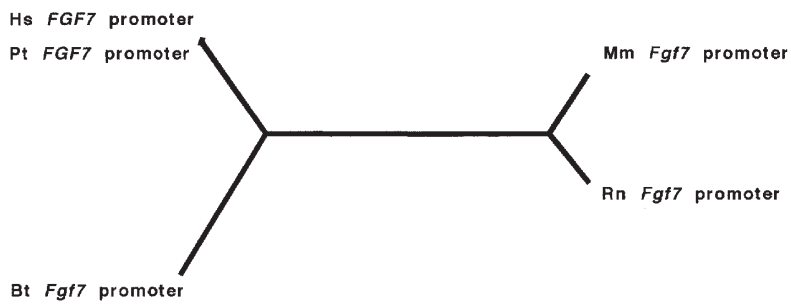

\section{B}

ATGATAGGATGTTTTCAGCTTTCTAGCTTTCAACAGATGCAATGATCAAAGAGTCTGTCCCCCTCTGCTTTAATAAATG GGAACATTCAACATTTGGCATATGAAGAGTAACTGGGTACATCTGAACTATTCTGTGCTCTTCATTCTGTTCCCTARAG $==m=m$

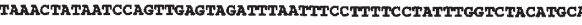
CCACCTGCOTCACATAGAGAGAGAGAGAGAGAGAGAGAGAGAGAGAGAGTGTGTGTGTGTGTG GTGTGCGCGCGCGCGCGTGCACGCGTGTETGTTTTGAGGAGTATATAACATGAAAAAGATAATPTTPATATACTTTCTTA ATACTGATTTAGATAGACAACACAATTATTCTACACCAAGAATCTAATACAACACTTGAGCACTACTTCATGTATAACAC AACAGGAAGTAAAGGAGAGAACTCATGCTGGAAAGTAAAATAGCTTTCACTPATTTACCTTAGCAATACATATTATAATT CCATCTCTAAATTAACATTTATTGTTCCAATATTCTTATTGAGGCAAAAATCAAACACTGATAATCAATGTGAAACCATY TCCTCCTITTTAAAGAATAACAGCTTPTAAAAGTAGGTGCCCTGTTCCTTATATTAGTAGTGAGTATAAGTCACAGATCA CAGAATTGTAAAGCACCATAGAGGCATTAGACCAATACACCAATTTTACAAATGTAGCAGCTGAGACTCTGGAACAGCAO Tran G crom

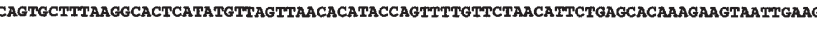
AAATGCATGTATCATAAAGCTATGGAAAAGATTTTCTAAGTATTAAAAAACCCTATATATATGTTCAAATGAATAATTTY CCAAGTATAATACAACTGCTATATTGCAAAGAAGGTTTAAAAAATTCCCTITCCCCTTCTAACTGCTTCAGAACCAGAGA TCTGTTTCTACAAAGTAGGTACCCACTTGTAGATTTAAGCATAAAACATGTTCATAGCTCCCTCTCATGACCATTGAATA CTATACAAGCCTCCTTTTATTCTCGCTGTTGTATTCAACTAACAGCTCACTGGCTAAGTTTTAATTGCTTCCAATGAGGT CAGCAAAGGTATTTATCGAAAAGCCCTGAATAAAAGGCTCACACACACACACAAGCACACACGCGCTCACACACAGAGAG

D

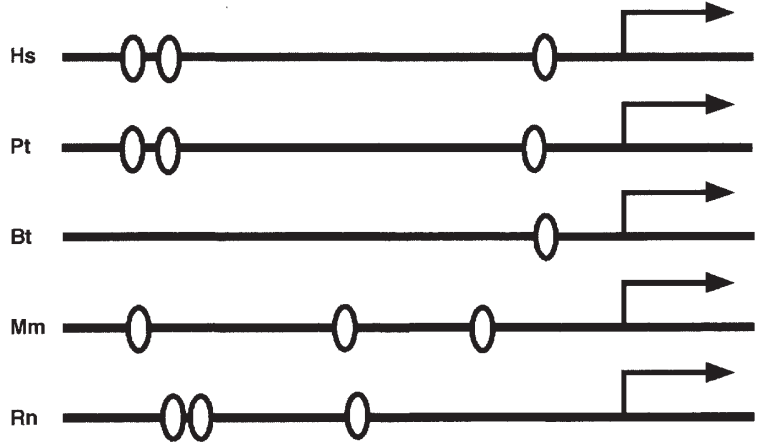

Figure 2. Comparative genomics on the $F G F$ family. (A), Human $F G F$ gene family. Gene symbol, RefSeq accession number, genome clone accession number, and TCF/LEF-binding sites within 5'-flanking promoter of $F G F$ family genes are listed. (B), Nucleotide sequence of human $F G F 7$ promoter. Region corresponding to exon 1 of human $F G F 7$ gene is boxed. Three TCF/LEF-binding sites are shown by double over-lines. (C), Phylogenetic analysis on vertebrate $F G F 7$ promoters. Cow $F g f 7$ promoter is more related to primate $F G F 7$ promoters than to rodent $F g f 7$ promoters. (D), Schematic representation of vertebrate $F G F 7$ promoters. Three TCF/LEF-binding sites within human FGF7 promoter are conserved in the chimpanzee FGF7 promoter, and the most proximal one in cow $F g f 7$ promoter, but not in rodent $F g f 7$ promoters.

promoter was conserved in the cow $F g f 7$ promoter, but not in rodent $F g f 7$ promoters (Fig. 2D). Because three TCF/LEFbinding sites within human FGF7 promoter were not conserved among mammals, it remained unclear whether human FGF7 is the target of the canonical WNT signaling pathway or not.

Expression analyses on FGF16, FGF 18 and FGF20 genes revealed that $F G F 18$ and $F G F 20$ genes are expressed in epithelial cells or cancer cells derived from gastrointestinal tract. Therefore, FGF1 8 and FGF20 are targets of the canonical WNT signaling pathway in the gastrointestinal tract.

\section{FGF signaling network in the stomach}

FGF2 and FGF7 are major FGFs implicated in embryogenesis and tissue regeneration within the stomach (31-33). FGF2 is expressed in submucosal tissues to transduce signals through FGFR1 expressed mucosal tissues. FGF7 is expressed in mesynchymal fibroblast to transduce signals through FGFR2 expressed in epithelial cells.

Helicobacter pylori is a causative pathogen for peptic ulcer diseases, chronic atrophic gastritis and gastric cancer (34-37, and refs. therein). FGF2 is one of the pro-angiogenic factors inducing healing of gastric mucosal damage associated with Helicobacter pylori infection. CagA protein and peptideglycan of Helicobacter pylori are injected into human gastric epithelial cells by using the Type IV secretion system. CagA protein is phosphorylated by SRC family protein kinases to activate SHP2 phosphatase. Because SHP2 is a component of docking protein complex for FGF signaling, SHP2 activation leads to FGF signaling activation (Fig. 3A).

Gastric cancer arises from normal tissues based on genetic predisposition for $\mathrm{CDH} 1$ gene, chronic Helicoabcter pylori infection, and salt over-uptake (34,38-40). Cancer cells are characterized by dysregulated proliferation, dysregulated adhesion, and also by resistance to apoptosis, senescence, and anti-cancer drugs. Cancer cells acquire malignant phenotypes mentioned above during multi-stage carcinogenesis due to the accumulation of multiple epigenetic changes and genetic alterations of cancer-associated genes. FGF7 secreted from mesenchymal fibroblasts stimulate the proliferation of gastric cancer, especially diffuse-type gastric cancer derived from fundic gland mucosa (41). FGFR2 gene is preferentially amplified in diffuse-type gastric cancer to overexpress FGFR2 splicing variant encoding a high-affinity FGF7 receptor (42). These facts clearly indicate that the FGF7 FGFR2 signaling cascade plays a critical role for the development and progression of diffuse-type gastric cancer.

\section{FGF signaling network in the colon}

FGF2 and FGF20 are major FGFs implicated in embryogenesis and tissue regeneration within the colon $(31,43,44)$. 
A

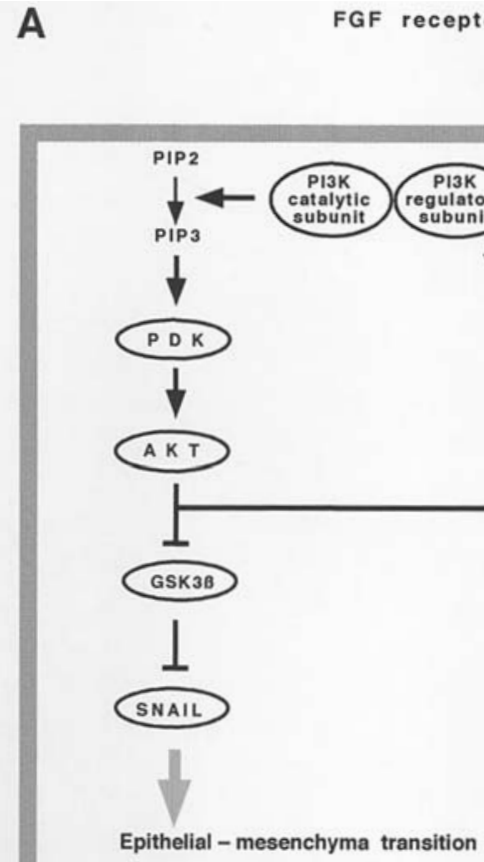

\section{.}

.
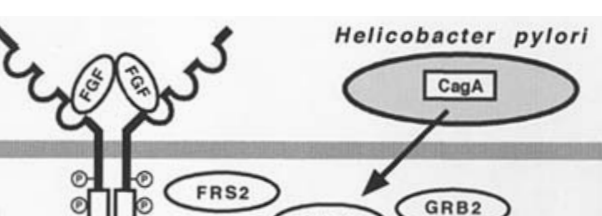

gubunity
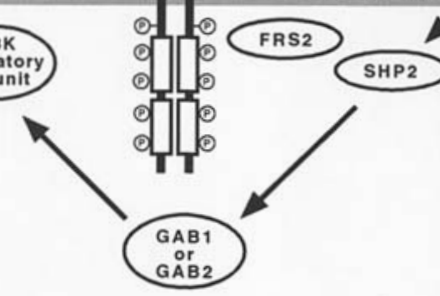

5
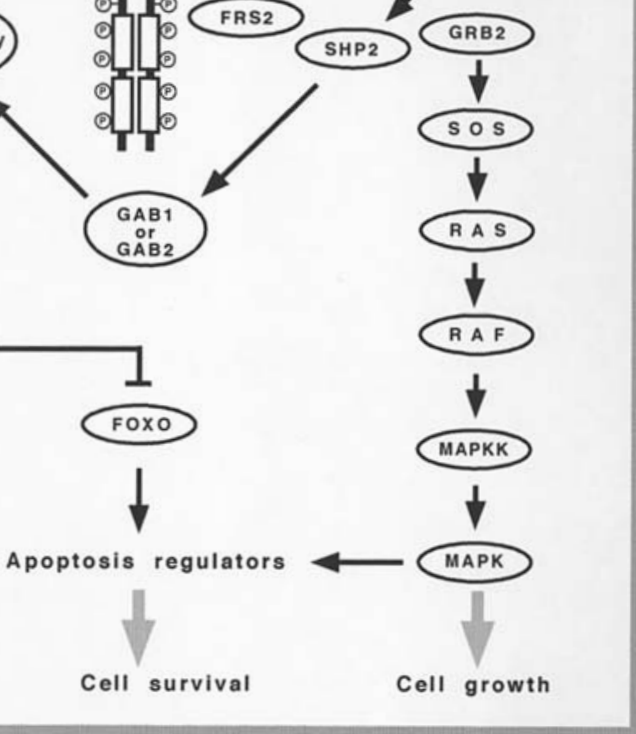

B

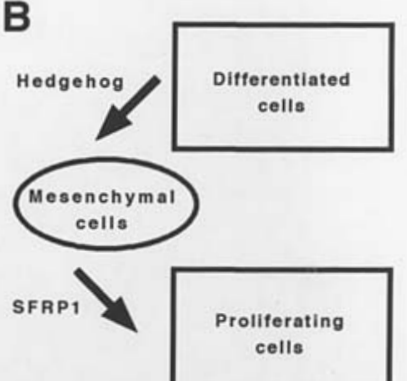

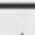

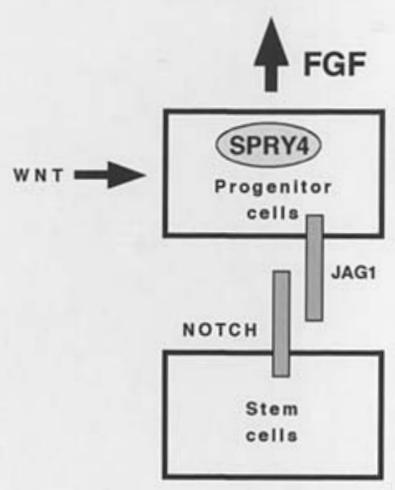

Figure 3. FGF signaling network in the gastrointestinal tract. (A), FGF signaling network during gastrocarcinogenesis. FGF7 secreted from mesenchymal fibroblasts activate FGF signaling through FGFR2 and FRS2-SHP2-GRB2 docking complex in gastric epithelial cells as well as in diffuse-type gastric cancer. Helicobacter pylori injects CagA protein and peptideglycan into human gastric epithelial cells by using Type IV secretion system. CagA phosphorylation by SRC family kinases leads to FGF signaling activation through SHP2 activation in precancerous lesion and early gastric cancer. FGFR2 overexpression associated with FGFR2 gene amplification leads to FGF signaling activation in advanced gastric cancer, especially in diffuse-type gastric cancer derived from fundic gland mucosa. (B), FGF signaling network in colorectal mucosa. Canonical WNT signaling activation in progenitor cells leads to transcriptional activation of SPRY4 and FGFs, which results in FGF signaling down-regulation in progenitor cells themselves as well as FGF signaling activation in proliferating cells. Canonical WNT signaling pathway is activated in progenitor cells, and FGF signaling pathway is activated in proliferating cells.

FGF2 is expressed in submucosal tissues to induce angiogenesis, while FGF20 is expressed in epithelial progenitor cells to induce epithelial proliferation.

We reported molecular cloning and characterization of human FGF20 in August, 2000 (43). Ohmachi et al claimed the same gene as a novel $F G F$ family member in October, 2000 (45), and Jeffers et al in April, 2001 (46). FGF20 mRNA is almost undetectable in normal human tissues by using Northern blot analyses, but is detected in SW480 colorectal cancer cells. FGF2O is upregulated in human colorectal cancer with the canonical WNT signaling activation.

SPROUTY (SPRY) and SPRED family members function as inhibitors for FGF signaling cascades. Among SPRY1, SPRY2, SPRY3, SPRY4, SPRED1, SPRED2, and SPRED3 genes, $S P R Y 4$ gene is the evolutionarily conserved target of the canonical WNT signaling pathway (47). WNT signaling activation in progenitor cells leads to growth regulation of progenitor cells themselves through SPRY4 induction, and also to growth stimulation of proliferating cells through FGF secretion (Fig. 3B). SPRY4 is the FGF signaling inhibitor functioning as negative feedback apparatus for the WNT/ FGF-dependent epithelial proliferation.

\section{Clinical application of FGF}

Recombinant human FGF7 (Palifermin) is clinically applied to decrease the incidence and duration of myelotoxic therapyinduced oral mucositis in cancer patient (48).
Recombinant human FGF20 (CG53135) is clinically applicable for mucosal restitution in patients with inflammatory bowel disease as well as for chemotherapy/radiation-induced oral mucositis in cancer patient $(44,49)$; however, the benefits of recombinant human FGF20 remain to be demonstrated by clinical trials.

Recombinant human FGF2 is clinically applicable for wound healing in patients with peptic ulcer or burn as well as for therapeutic angiogenesis in patients with cardiovascular diseases (50); however, the benefit of recombinant human FGF2 is not significant in the general population.

Adenovirus containing human FGF4 (GENERX) developed by Collateral Therapeutics and Shering AG might be applicable for mucosal restitution in patients with inflammatory bowel disease or radiation-induced colitis as well as for therapeutic angiogenesis in patients with cardiovascular diseases (51); however, the benefits of adenovirus containing human FGF4 remain to be demonstrated by clinical trials.

\section{Clinical application of FGF signaling inhibitors}

Small-molecule inhibitors and antibody drugs targeted to growth factor signaling molecules appeared as novel therapeutic agents for cancer patients in the 21st century. Gefitinib (Iressa), Erlotinib (Tarceva) and Lapatinib (GW572016) are small molecule inhibitors for receptors of EGF family members, while Cetuximab (Erbitux), Pertuzumab (Omnitarg) and Trastuzumab (Herceptin) are monoclonal antibodies targeted to EGF family ligands or receptors (52-54). 
In silico screening of ATP mimetic compounds, fitting into ATP-binding cassette of protein kinases, are performed in the post-genome era to facilitate the efficacy of high-throughput screening for protein kinase inhibitors by using threedimensional structure of protein kinases and small-molecule compounds for docking simulation software. PD173074 is a small-molecule inhibitor for FGFR, while RO4396686 and SU6668 are small-molecule inhibitors for FGFR and other tyrosine kinases (55-57).

\section{Perspectives}

Epigenetic changes occur at the early stage during multi-stage carcinogenesis associated with chronic persistent inflammation and/or aging (58). SFRP1 gene, encoding WNT antagonist, is silenced due to promoter $\mathrm{CpG}$ hypermethylation in gastrointestinal cancers. HHIP1 (HHIP) gene, encoding Hedgehog antagonist, is also silenced due to promoter $\mathrm{CpG}$ hypermethylation in gastrointestinal cancers. Because SPRY and $S P R E D$ family genes encode FGF signaling inhibitors, promoter CpG hypermethylation of SPRY and SPRED family genes in gastrointestinal cancers should be searched for in the future.

Small-molecule inhibitors for FGFR are promising anticancer drugs for certain patients with diffuse type gastric cancer. Single nucleotide polymorphisms (SNPs) or copy number polymorphism (CNP) analyses on FGF signaling genes as well as transcriptome or proteome analyses on FGF family ligands could lead to the establishment of criteria to predict which patients should be treated with small-molecule FGFR inhibitors.

Although small-molecule inhibitors are initially effective for some cancer patients, recurrence due to drug resistance associated with somatic mutations of drug-target genes occurs in most cases. Cocktail therapy using multiple protein kinase inhibitors could enhance the therapeutic effects for gastrointestinal cancer through the reduction of recurrence associated with somatic mutations.

Genetic predisposition, chronic persistent inflammation, life style and aging are causative factors for gastrointestinal cancers. Comprehensive characterization of host genome as well as bacterial genomes could promote the realization of personalized prevention and personalized medicine to improve the prognosis of cancer patients.

\section{References}

1. Katoh M: $W N T$ and $F G F$ gene clusters. Int J Oncol 21: 1269-1273, 2002.

2. Katoh M and Katoh M: Bioinformatics for cancer management in the post-genome era. Technol Cancer Res Treat 5: 169-176, 2006.

3. Eswarakumar VP, Lax I and Schlessinger J: Cellular signaling by fibroblast growth factor receptors. Cytokine Growth Factor Rev 16: 139-149, 2005.

4. Dailey L, Ambrosetti D, Mansukhani A and Basilico C: Mechanisms underlying differential responses to FGF signaling. Cytokine Growth Factor Rev 16: 233-247, 2005.

5. Katoh M and Katoh M: Evolutionary conservation of CCND1ORAOV1-FGF 19-FGF4 locus from zebrafish to human. Int $\mathbf{J}$ Mol Med 12: 45-50, 2003.

6. Fischer S, Draper BW and Neumann CJ: The zebrafish $f g f 24$ mutant identifies an additional level of Fgf signaling involved in vertebrate forelimb initiation. Development 130: 3515-3524, 2003.

7. Katoh Y and Katoh M: Comparative genomics on FGF7, FGF10, $F G F 22$ orthologs, and identification of $f g f 25$. Int J Mol Med 16: 767-770, 2005 .
8. Shackleford GM, MacArthur CA, Kwan HC and Varmus HE: Mouse mammary tumor virus infection accelerates mammary carcinogenesis in Wnt-1 transgenic mice by insertional activation of int-2/Fgf-3 and Hst/Fgf-4. Proc Natl Acad Sci USA 90: 740-744, 1993.

9. Lee FS, Lane TF, Kuo A, Shackleford GM and Leder P: Insertional mutagenesis identifies a member of the Wnt gene family as a candidate oncogene in the mammary epithelium of int-2/Fgf-3 transgenic mice. Proc Natl Acad Sci USA 92: 2268-2272, 1995.

10. Katoh M, Hirai M, Sugimura T and Terada M: Cloning, expression and chromosomal localization of WNT13, a novel member of the WNT gene family. Oncogene 13: 873-876, 1996.

11. Saitoh $\mathrm{T}$ and Katoh M: Molecular cloning and characterization of human WNT8A. Int J Oncol 19: 123-127, 2001.

12. Katoh M: Frequent up-regulation of WNT2 in primary gastric cancer and colorectal cancer. Int J Oncol 19: 1003-1007, 2001.

13. Kirikoshi H, Sekihara $\mathrm{H}$ and Katoh M: WNT10A and WNT6, clustered in human chromosome $2 \mathrm{q} 35$ region with head to tail manner, are strongly co-expressed in SW480 cells. Biochem Biophys Res Commun 283: 798-805, 2001.

14. Saitoh T, Hirai $M$ and Katoh M: Molecular cloning and characterization of WNT3A and WNT14 clustered in human chromosome 1q42 region. Biochem Biophys Res Commun 284: 1168-1175, 2001

15. Saitoh T, Mine T and Katoh M: Up-regulation of WNT $8 B$ mRNA in human gastric cancer. Int J Oncol 20: 343-348, 2002.

16. Katoh M: Regulation of WNT signaling molecules by retinoic acid during neuronal differentiation in NT2 cells: threshold model of WNT action. Int J Mol Med 10: 683-687, 2002.

17. Katoh $\mathrm{M}$ and Katoh $\mathrm{M}$ : Identification and characterization of human BCL9L gene and mouse Bcl9l gene in silico. Int J Mol Med 12: 643-649, 2003.

18. Heller RS, Klein T, Ling Z, Heimberg H, Katoh M, Madsen OD and Serup P: Expression of WNT, Frizzled, sFRP, and DKK genes in adult human pancreas. Gene Expr 11: 141-147, 2003.

19. Garciadiego-Cazares D, Rosales C, Katoh M and ChimalMonroy J: Coordination of chondrocyte differentiation and joint formation by $\alpha 5 \beta 1$ integrin in the developing appendicular skeleton. Development 131: 4735-4742, 2004.

20. Katoh M: WNT2B: comparative integromics and clinical application. Int J Mol Med 16: 1103-1108, 2005.

21. Swain RK, Katoh M, Medina A and Steinbeisser H: Xenopus frizzled-4S, a splicing variant of Xfz4, is a context-dependent activator and inhibitor of Wnt/B-catenin signaling. Cell Commun Signal 3: 12, 2005.

22. Katoh $\mathrm{Y}$ and Katoh M: Comparative genomics on FGF16 orthologs. Int J Mol Med 16: 959-963, 2005.

23. Katoh M and Katoh M: Comparative genomics on $F G F 8$, FGF17, and FGF18 orthologs. Int J Mol Med 16: 493-496, 2005.

24. Katoh $\mathrm{M}$ and Katoh M: Comparative genomics on FGF20 orthologs. Oncol Rep 14: 287-290, 2005.

25. Katoh M: Paradigm shift in gene-finding method: from benchtop approach to desk-top approach. Int J Mol Med 10: 677-682, 2002.

26. Katoh Y and Katoh M: Comparative genomics on DKK1 orthologs. Int J Oncol 27: 275-279, 2005.

27. Katoh $\mathrm{Y}$ and Katoh M: Comparative genomics on $D K K 2$ and DKK4 orthologs. Int J Mol Med 16: 477-481, 2005.

28. Katoh Y and Katoh M: WNT antagonist, SFRP1, is Hedgehog signaling target. Int J Mol Med 17: 171-175, 2006.

29. Katoh Y and Katoh M: Comparative genomics on HHIP family orthologs. Int J Mol Med 17: 391-395, 2006.

30. Katoh M and Katoh M: Notch ligand, JAG1, is evolutionarily conserved target of canonical WNT signaling pathway in progenitor cells. Int J Mol Med 17: 681-685, 2006.

31. Gonzalez AM, Hill DJ, Logan A, Maher PA and Baird A: Distribution of fibroblast growth factor (FGF)-2 and FGF receptor-1 messenger RNA expression and protein presence in the mid-trimester human fetus. Pediatr Res 39: 375-385, 1996.

32. Hull MA, Brough JL, Powe DG, et al: Expression of basic fibroblast growth factor in intact and ulcerated human gastric mucosa. Gut 43: 525-536, 1998.

33. Kinoshita Y, Nakata H, Hassan $\mathrm{S}$, et al: Gene expression of keratinocyte and hepatocyte growth factors during the healing of rat gastric mucosal lesions. Gastroenterology 109: 1068-1077, 1995 . 
34. Katoh M and Terada M: Oncogenes and tumor suppressor genes. In: Gastric Cancer. Nishi M, et al (eds). Springer-Verlag, Tokyo, pp196-208, 1993

35. Peek RM Jr and Crabtree JE: Helicobacter pylori infection and gastric neoplasia. J Pathol 208: 233-248, 2006.

36. Jones MK, Tomikawa M, Mohajer B and Tarnawski AS: Gastrointestinal mucosal regeneration: role of growth factors. Front Biosci 4: D303-D309, 1999.

37. Yoshida N, Ishikawa T, Ichiishi E, et al: The effect of rebamipide on Helicobacter pylori extract-mediated changes of gene expression in gastric epithelial cells. Aliment Pharmacol Ther 18 (Suppl 1): 63-75, 2003.

38. Katoh M and Katoh M: Pharmacogenomics on gastric cancer. Cancer Biol Ther 3: 566-567, 2004

39. Katoh $\mathrm{Y}$ and Katoh M: Hedgehog signaling in gastric cancer. Cancer Biol Ther 4: 1050-1054, 2005.

40. Katoh M: Epithelial-mesenchymal transition in gastric cancer. Int J Oncol 27: 1677-1683, 2005.

41. Nakazawa K, Yashiro M and Hirakawa K: Keratinocyte growth factor produced by gastric fibroblasts specifically stimulates proliferation of cancer cells from scirrhous gastric carcinoma. Cancer Res 63: 8848-8852, 2003.

42. Katoh M, Itoh H, Ishii H, et al: Implication of the K-sam gene in the develpoment and progression of gastric cancer. In: 1st International Gastric Cancer Congress. Nishi M, et al (eds). Monduzzi Editore, Bologna, pp763-766, 1995.

43. Kirikoshi H, Sagara N, Saitoh T, Tanaka K, Sekihara H, Shiokawa K and Katoh M: Molecular cloning and characterization of human FGF20 on chromosome 8p21.3-p22. Biochem Biophys Res Commun 274: 337-343, 2000.

44. Jeffers M, McDonald WF, Chillakuru RA, et al: A novel human fibroblast growth factor treats experimental intestinal inflammation. Gastroenterology 123: 1151-1162, 2002.

45. Ohmachi S, Watanabe Y, Mikami T, Kusu N, Ibi T, Akaike A and Itoh N: FGF-20, a novel neurotrophic factor, preferentially expressed in the substantia nigra pars compacta of rat brain Biochem Biophys Res Commun 277: 355-360, 2000.
46. Jeffers M, Shimkets R, Prayaga S, et al: Identification of a novel human fibroblast growth factor and characterization of its role in oncogenesis. Cancer Res 61: 3131-3138, 2001.

47. Katoh Y and Katoh M: FGF signaling inhibitor, SPRY4, is evolutionarily conserved target of WNT signaling pathway in progenitor cells. Int J Mol Med 17: 529-532, 2006.

48. Siddiqui MA and Wellington K: Palifermin: in myelotoxic therapy-induced oral mucositis. Drugs 65: 2139-2146, 2005.

49. Alvarez E, Fey EG, Valax P, et al: Preclinical characterization of CG53135 (FGF-20) in radiation and concomitant chemotherapy/radiation-induced oral mucositis. Clin Cancer Res 9: 3454-3461, 2003

50. Aviles RJ, Annex BH and Lederman RJ: Testing clinical therapeutic angiogenesis using basic fibroblast growth factor (FGF-2). Br J Pharmacol 140: 637-646, 2003.

51. Collateral Therapeutics: FGF-4 gene therapy GENERX. BioDrugs 16: 75-76, 2002

52. Reichert JM, Rosensweig CJ, Faden LB and Dewitz MC: Monoclonal antibody success in the clinic. Nat Biotechnol 23: 1073-1078, 2005.

53. Krause DS and van Etten RA: Tyrosine kinases as targets for cancer therapy. N Engl J Med 353: 172-187, 2005.

54. Garber K: The second wave in kinase cancer drugs. Nat Biotechnol 24: 127-130, 2006.

55. Ezzat S, Huang P, Dackiw A and Asa SL: Dual inhibition of RET and FGFR4 restrains medullary thyroid cancer cell growth. Clin Cancer Res 11: 1336-1341, 2005.

56. McDermott LA, Higgins B, Simcox M, et al: Biological evaluation of a multi-targeted small molecule inhibitor of tumorinduced angiogenesis. Bioorg Med Chem Lett 16: 1950-1953, 2006.

57. Sessa C, Vigano L, Grasselli G, et al: Phase I clinical and pharmacological evaluation of the multi-tyrosine kinase inhibitor SU006668 by chronic oral dosing. Eur J Cancer 42: 171-178, 2006.

58. Baylin SB and Ohm JE: Epigenetic gene silencing in cancer - a mechanism for early oncogenic pathway addiction? Nat Rev Cancer 6: 107-116, 2006. 